\title{
Improved overall survival in melanoma with combined dabrafenib and trametinib
}

Robert, Caroline ; Karaszewska, Boguslawa ; Schachter, Jacob ; Rutkowski, Piotr ; Mackiewicz, Andrzej ; Stroiakovski, Daniil ; Lichinitser, Michael ; Dummer, Reinhard ; Grange, Florent ; Mortier, Laurent ; Chiarion-Sileni, Vanna ; Drucis, Kamil ; Krajsova, Ivana ; Hauschild, Axel ; Lorigan, Paul ; Wolter,

Pascal ; Long, Georgina V ; Flaherty, Keith ; Nathan, Paul ; Ribas, Antoni ; Martin, Anne-Marie ; Sun, Peng ; Crist, Wendy ; Legos, Jeff ; Rubin, Stephen D ; Little, Shonda M ; Schadendorf, Dirk

\begin{abstract}
BACKGROUND The BRAF inhibitors vemurafenib and dabrafenib have shown efficacy as monotherapies in patients with previously untreated metastatic melanoma with BRAF V600E or V600K mutations. Combining dabrafenib and the MEK inhibitor trametinib, as compared with dabrafenib alone, enhanced antitumor activity in this population of patients. METHODS In this open-label, phase 3 trial, we randomly assigned 704 patients with metastatic melanoma with a BRAF V600 mutation to receive either a combination of dabrafenib (150 mg twice daily) and trametinib (2 $\mathrm{mg}$ once daily) or vemurafenib (960 mg twice daily) orally as first-line therapy. The primary end point was overall survival. RESULTS At the preplanned interim overall survival analysis, which was performed after $77 \%$ of the total number of expected events occurred, the overall survival rate at 12 months was $72 \%$ (95\% confidence interval [CI], 67 to 77$)$ in the combination-therapy group and $65 \%$ (95\% CI, 59 to 70$)$ in the vemurafenib group (hazard ratio for death in the combination-therapy group, $0.69 ; 95 \% \mathrm{CI}, 0.53$ to $0.89 ; \mathrm{P}=0.005$ ). The prespecified interim stopping boundary was crossed, and the study was stopped for efficacy in July 2014. Median progression-free survival was 11.4 months in the combination-therapy group and 7.3 months in the vemurafenib group (hazard ratio, $0.56 ; 95 \% \mathrm{CI}, 0.46$ to $0.69 ; \mathrm{P}<0.001$ ). The objective response rate was $64 \%$ in the combination-therapy group and $51 \%$ in the vemurafenib group $(\mathrm{P}<0.001)$. Rates of severe adverse events and study-drug discontinuations were similar in the two groups. Cutaneous squamous-cell carcinoma and keratoacanthoma occurred in $1 \%$ of patients in the combination-therapy group and $18 \%$ of those in the vemurafenib group. CONCLUSIONS Dabrafenib plus trametinib, as compared with vemurafenib monotherapy, significantly improved overall survival in previously untreated patients with metastatic melanoma with BRAF V600E or V600K mutations, without increased overall toxicity. (Funded by GlaxoSmithKline; ClinicalTrials.gov number, NCT01597908.).
\end{abstract}

DOI: https://doi.org/10.1056/NEJMoa1412690

Posted at the Zurich Open Repository and Archive, University of Zurich

ZORA URL: https://doi.org/10.5167/uzh-107163

Journal Article

Published Version

Originally published at:

Robert, Caroline; Karaszewska, Boguslawa; Schachter, Jacob; Rutkowski, Piotr; Mackiewicz, Andrzej; Stroiakovski, Daniil; Lichinitser, Michael; Dummer, Reinhard; Grange, Florent; Mortier, Laurent; Chiarion- 
Sileni, Vanna; Drucis, Kamil; Krajsova, Ivana; Hauschild, Axel; Lorigan, Paul; Wolter, Pascal; Long, Georgina V; Flaherty, Keith; Nathan, Paul; Ribas, Antoni; Martin, Anne-Marie; Sun, Peng; Crist, Wendy; Legos, Jeff; Rubin, Stephen D; Little, Shonda M; Schadendorf, Dirk (2015). Improved overall survival in melanoma with combined dabrafenib and trametinib. New England Journal of Medicine, 372(1):30-39.

DOI: https://doi.org/10.1056/NEJMoa1412690 


\title{
Improved Overall Survival in Melanoma with Combined Dabrafenib and Trametinib
}

\author{
Caroline Robert, M.D., Ph.D., Boguslawa Karaszewska, M.D., Jacob Schachter, M.D., \\ Piotr Rutkowski, M.D., Ph.D., Andrzej Mackiewicz, M.D., Ph.D., \\ Daniil Stroiakovski, M.D., Michael Lichinitser, M.D., Reinhard Dummer, M.D., \\ Florent Grange, M.D., Ph.D., Laurent Mortier, M.D., Vanna Chiarion-Sileni, M.D., \\ Kamil Drucis, M.D., Ph.D., Ivana Krajsova, M.D., Axel Hauschild, M.D., Ph.D., \\ Paul Lorigan, M.D., Pascal Wolter, M.D., Georgina V. Long, M.D., Ph.D., \\ Keith Flaherty, M.D., Paul Nathan, M.D., Ph.D., Antoni Ribas, M.D., Ph.D., \\ Anne-Marie Martin, Ph.D., Peng Sun, Ph.D., Wendy Crist, B.A., Jeff Legos, Ph.D., \\ Stephen D. Rubin, M.D., Shonda M. Little, M.P.H., and Dirk Schadendorf, M.D.
}

\begin{abstract}
The authors' affiliations are listed in the Appendix. Address reprint requests to Dr. Robert at the Dermatology Service and INSERM Unité 981 , Gustave Roussy, 114 rue Edouard Vaillant, 94805 VillejuifParis Sud, France, or at caroline.robert@ gustaveroussy.fr.
\end{abstract}

This article was published on November 16, 2014, at NEJM.org.

N Engl J Med 2015;372:30-9. DOI: $10.1056 /$ NEJMoal412690

Copyright (C) 2014 Massachusetts Medical Society.

\section{A BSTRACT}

\section{BACKGROUND}

The BRAF inhibitors vemurafenib and dabrafenib have shown efficacy as monotherapies in patients with previously untreated metastatic melanoma with BRAF V600E or V600K mutations. Combining dabrafenib and the MEK inhibitor trametinib, as compared with dabrafenib alone, enhanced antitumor activity in this population of patients.

\section{METHODS}

In this open-label, phase 3 trial, we randomly assigned 704 patients with metastatic melanoma with a BRAF V600 mutation to receive either a combination of dabrafenib (150 mg twice daily) and trametinib (2 mg once daily) or vemurafenib (960 mg twice daily) orally as first-line therapy. The primary end point was overall survival.

\section{RESULTS}

At the preplanned interim overall survival analysis, which was performed after $77 \%$ of the total number of expected events occurred, the overall survival rate at 12 months was $72 \%$ (95\% confidence interval [CI], 67 to 77 ) in the combination-therapy group and $65 \%$ (95\% CI, 59 to 70 ) in the vemurafenib group (hazard ratio for death in the combination-therapy group, $0.69 ; 95 \% \mathrm{CI}, 0.53$ to $0.89 ; \mathrm{P}=0.005)$. The prespecified interim stopping boundary was crossed, and the study was stopped for efficacy in July 2014. Median progression-free survival was 11.4 months in the combinationtherapy group and 7.3 months in the vemurafenib group (hazard ratio, 0.56 ; $95 \%$ CI, 0.46 to 0.69 ; $\mathrm{P}<0.001$ ). The objective response rate was $64 \%$ in the combinationtherapy group and $51 \%$ in the vemurafenib group $(\mathrm{P}<0.001)$. Rates of severe adverse events and study-drug discontinuations were similar in the two groups. Cutaneous squamous-cell carcinoma and keratoacanthoma occurred in 1\% of patients in the combination-therapy group and $18 \%$ of those in the vemurafenib group.

\section{CONCLUSIONS}

Dabrafenib plus trametinib, as compared with vemurafenib monotherapy, significantly improved overall survival in previously untreated patients with metastatic melanoma with BRAF V600E or V600K mutations, without increased overall toxicity. (Funded by GlaxoSmithKline; ClinicalTrials.gov number, NCT01597908.) 
T HE TREATMENT OF METASTATIC MELAnoma is rapidly evolving. The potent and specific BRAF inhibitors vemurafenib and dabrafenib, as compared with chemotherapy, have significantly improved response rates, along with progression-free and overall survival, in patients with metastatic melanoma with BRAF V600E or V600K mutations. ${ }^{1,2}$ However, acquired resistance to BRAF inhibitors frequently develops through reactivation of the mitogen-activated protein kinase (MAPK) pathway, resulting in a median progression-free survival of 6 to 8 months. ${ }^{2-5}$ In addition, the use of BRAF inhibitors may result in the development of secondary skin tumors, originating from a paradoxical activation of the MAPK pathway in cells without a BRAF mutation. ${ }^{1,6-11}$ Combining a BRAF inhibitor with a MEK inhibitor addresses the limitations of single-agent BRAF inhibitors and results in a significant delay in the emergence of resistance, with a longer median progression-free survival than with dabrafenib alone, as well as a decreased incidence of BRAFinhibitor-induced skin tumors. ${ }^{12-14}$

In this open-label, randomized, phase 3 study, we evaluated the effect of combination therapy with dabrafenib plus trametinib versus vemurafenib monotherapy on overall survival in previously untreated patients with unresectable stage IIIC or IV melanoma with BRAF V600E or V600K mutations.

\section{METHODS}

\section{PATIENTS}

From June 2012 through October 2013, a total of 1645 patients were screened at 193 centers worldwide. The presence of BRAF V600E or V600K mutations was centrally determined with the investigational use of the THxID BRAF assay (bioMérieux). Additional key eligibility criteria were measureable disease, according to the Response Evaluation Criteria in Solid Tumors (RECIST), version 1.1, ${ }^{15}$ and an Eastern Cooperative Oncology Group (ECOG) performance status of 0 or 1 (on a scale of 0 to 5 , with 0 indicating no symptoms and higher numbers reflecting greater disability). ${ }^{16}$ Patients who had undergone treatment for brain metastases with no increase in lesion size for at least 12 weeks were eligible for enrollment. Additional eligibility criteria are provided in the Methods section in the Supplementary Appendix, available with the full text of this article at NEJM.org. Written informed consent was obtained from all study participants.

\section{STUDY DESIGN AND TREATMENT}

Eligible patients were assigned in a $1: 1$ ratio to receive either a combination of dabrafenib (150 mg orally twice daily) and trametinib (2 mg orally once daily) or vemurafenib (960 mg orally twice daily). The primary end point was overall survival. Secondary end points included progression-free survival, overall response rate, duration of response, and safety. Crossover was prohibited until the independent data and safety monitoring committee recommended stopping the study early for efficacy. After the recommendation, the study protocol was amended to allow patients in the vemurafenib group to cross over to the combination-therapy group.

\section{ASSESSMENT}

We conducted tumor assessments according to RECIST, version $1.1,{ }^{15}$ at baseline, every 8 weeks until week 56, and then every 12 weeks until disease progression, death, or withdrawal from the study (Table S1 in the Supplementary Appendix). All responses were confirmed with a scan at least 4 weeks after the first RECIST response. Adverse events were graded by the investigator, according to the National Cancer Institute Common Terminology Criteria for Adverse Events, version 4.0, until day 30 after the discontinuation of study treatment.

\section{STUDY OVERSIGHT}

The study was funded by the sponsor, GlaxoSmithKline, and conducted in accordance with the provisions of the Declaration of Helsinki and Good Clinical Practice guidelines. The protocol was approved by the institutional review board or human research ethics committee at each study center. Data were collected at each study site and monitored by the sponsor. The first author wrote the first draft of the manuscript, with support from the last author and representatives of the sponsor. Representatives of the sponsor designed the study, confirmed the accuracy of the data, and compiled the data for analysis. All the authors and sponsor representatives had full access to the study data and were involved in their analysis. No one who was not an author contributed to the writing of the manuscript. Editorial assistance that did not involve writing was provided by SciMentum and funded by the sponsor. All the authors vouch for the accuracy and completeness of the data reported and for the fidelity of the study to the protocol (available at NEJM.org). 


\section{STATISTICAL ANALYSIS}

We used the Kaplan-Meier method to estimate overall and progression-free survival. We evaluated between-group comparisons using a logrank test that was stratified for the BRAF mutation status (V600E vs. V600K) and the baseline level of lactate dehydrogenase (LDH) (above the upper limit of the normal range vs. the upper limit of the normal range or less). We estimated that 288 events would be required to detect a hazard ratio for death of 0.675 with an alpha level of 0.05 (i.e., an increase in median overall survival from 13.5 months in the vemurafenib group to 20 months in the combination-therapy group). Overall survival was defined as the time from randomization until death from any cause.

A preplanned interim analysis for overall survival was to be conducted when 202 of 288 events that were required for the final analysis (70\%) had been observed. Owing to the inherent lag in data entry, the actual number of deaths was 222 at the time of the interim analysis. Per protocol, efficacy boundaries were adjusted on the basis of the actual number of deaths at the time of the interim analysis. The data and safety monitoring committee used the adjusted stopping boundaries (two-sided $\mathrm{P}<0.0214$ for the efficacy analysis and $\mathrm{P}>0.2210$ for the futility analysis) to review the interim data. The committee recommended stopping for efficacy. As such, the interim summary is considered to be the final analysis of overall survival.

\section{RESULTS}

\section{PATIENTS}

Of the 1645 patients who underwent screening at 193 centers, 704 underwent randomization, with 352 patients in each group. A total of 68 patients (10\%, 34 in each study group) had a BRAF V600K mutation. Baseline characteristics of the patients are provided in Table 1. Known prognostic measures were well balanced in the two groups except for sex $(59 \%$ men in the combination-therapy group vs. $51 \%$ in the vemurafenib group). At the date of the analysis, the median follow-up durations were 11 months and 10 months and the median exposure durations were 10 months and 6 months in the combination-therapy group and the vemurafenib group, respectively.

A total of 80 patients in the combinationtherapy group and 81 in the vemurafenib group
(23\% in each group) continued to receive study treatment for at least 15 days after disease progression, according to the protocol, which allowed for treatment after progression on the basis of a request from an investigator for patients who seemed to benefit from the treatment in spite of RECIST progression. The median duration of study treatment after progression was similar in the two study groups and was less than 3 months for the majority of patients, with more patients continuing to receive the combination therapy for 6 to 12 months (9\%), as compared with those who continued to receive vemurafenib (1\%) (Table S2 in the Supplementary Appendix).

\section{EFFICACY}

The interim analysis for overall survival was performed in the intention-to-treat population of 352 patients in each group. At the data-cutoff date of April 17, 2014, the interim analysis was performed after 222 events had occurred. For the overall survival analysis, 100 patients (28\%) in the combination-therapy group and 122 (35\%) in the vemurafenib group had died (hazard ratio for death in the combination-therapy group, 0.69; $95 \%$ confidence interval [CI], 0.53 to 0.89 ; $\mathrm{P}=0.005)$ (Fig. 1A). The prespecified stopping boundary $(\mathrm{P}<0.0214)$ was crossed, and the study was stopped for efficacy on July 14, 2014. A protocol amendment was issued to allow crossover to the combination-therapy group for patients assigned to the vemurafenib group, but no patient had crossed over as of the effective datafreezing date of June 27, 2014.

The median overall survival was 17.2 months for patients in the vemurafenib group and had not been reached for patients in the combination-therapy group (Fig. 1A). The rate of overall survival at 12 months was $72 \%$ (95\% CI, 67 to 77 ) in the combination-therapy group and $65 \%$ ( $95 \%$ CI, 59 to 70 ) in the vemurafenib group. The hazard ratios for the variables of V600 mutation type, sex, age ( $<65$ years vs. $\geq 65$ years), baseline LDH level, and ECOG status have been calculated, although these subgroup analyses were not powered to show a significant between-group difference. They all favored the combination-therapy group except for the subgroup of patients with an ECOG score of 1 (hazard ratio, 1.03) (Fig. 1B).

Median progression-free survival was longer in the combination-therapy group than in the vemurafenib group (11.4 months vs. 7.3 months; 


\begin{tabular}{|c|c|c|c|}
\hline Characteristic & $\begin{array}{c}\text { Dabrafenib plus } \\
\text { Trametinib } \\
\text { (N=352) }\end{array}$ & $\begin{array}{l}\text { Vemurafenib } \\
(\mathrm{N}=352)\end{array}$ & $\begin{array}{l}\text { All Patients } \\
(\mathrm{N}=704)\end{array}$ \\
\hline Median age (range) $-\mathrm{yr}$ & $55(18-91)$ & $54(18-88)$ & $55(18-91)$ \\
\hline Male sex — no. (\%) & $208(59)$ & $180(51)$ & $388(55)$ \\
\hline Previous immunotherapy — no. (\%) $†$ & $61(17)$ & $93(26)$ & $154(22)$ \\
\hline \multicolumn{4}{|l|}{ ECOG score - no./total no. (\%) } \\
\hline 0 & $248 / 350(71)$ & $248 / 352(70)$ & $496 / 702(71)$ \\
\hline 1 & $102 / 350(29)$ & $104 / 352(30)$ & $206 / 702(29)$ \\
\hline \multicolumn{4}{|l|}{ BRAF mutation - no./total no. $(\%) \ddagger$} \\
\hline V600E & $312 / 346(90)$ & $317 / 351(90)$ & $629 / 697(89)$ \\
\hline V600K & $34 / 346(10)$ & $34 / 351(10)$ & $68 / 697(10)$ \\
\hline \multicolumn{4}{|l|}{$\begin{array}{l}\text { Tumor stage at screening - no./total } \\
\text { no. }(\%)\end{array}$} \\
\hline IVMlc & $221 / 351(63)$ & $208 / 351(59)$ & $429 / 702(61)$ \\
\hline IIIc, IVMla, or IVMlb & $130 / 351(37)$ & $143 / 351(41)$ & $273 / 702$ (39) \\
\hline \multicolumn{4}{|l|}{$\begin{array}{l}\text { Metastasis stage at screening - no./total } \\
\text { no. (\%) }\end{array}$} \\
\hline Mo & $14 / 351(4)$ & $26 / 351(7)$ & $40 / 702(6)$ \\
\hline $\mathrm{Mla}$ & $55 / 351(16)$ & $50 / 351(14)$ & $105 / 702(15)$ \\
\hline $\mathrm{Mlb}$ & $61 / 351(17)$ & $67 / 351(19)$ & $128 / 702(18)$ \\
\hline Mlc & $221 / 351(63)$ & $208 / 351(59)$ & $429 / 702(61)$ \\
\hline \multicolumn{4}{|l|}{ Baseline LDH - no./total no. (\%) } \\
\hline Above ULN & $118 / 351(34)$ & $114 / 352(32)$ & $232 / 703(33)$ \\
\hline ULN or less & $233 / 351(66)$ & $238 / 352(68)$ & $471 / 703(67)$ \\
\hline \multicolumn{4}{|l|}{$\begin{array}{l}\text { Visceral disease at baseline - no./total } \\
\text { no. (\%) } \int\end{array}$} \\
\hline Yes & 278/351 (79) & $271 / 352(77)$ & $549 / 703(78)$ \\
\hline No & $73 / 351(21)$ & $81 / 352(23)$ & $154 / 703(22)$ \\
\hline \multicolumn{4}{|c|}{$\begin{array}{l}\text { Number of disease sites at baseline }- \text { no./ } \\
\text { total no. (\%)\| }\end{array}$} \\
\hline Fewer than 3 & $177 / 351(50)$ & $201 / 352(57)$ & $378 / 703(54)$ \\
\hline 3 or more & $174 / 351(50)$ & $151 / 352(43)$ & $325 / 703(46)$ \\
\hline
\end{tabular}

* Data are missing in several categories for one patient in each study group because data were either missing or incorrect at baseline. There were no significant differences between the groups at baseline except for sex $(P=0.03)$. ECOG denotes Eastern Cooperative Oncology Group, LDH lactate dehydrogenase, and ULN upper limit of the normal range. $\uparrow$ Previous immunotherapy included interferon-alfa, interferon-gamma, interleukin-2, granulocyte-macrophage colony-stimulating factor, gangliosides, imiquimod, ipilimumab, and investigational antineoplastic vaccine. Interferon and interleukin were classified as biologics in the clinical database. They were reclassified as immunotherapy in this ad hoc analysis.

$¥$ Six patients in the combination-therapy group and one in the vemurafenib group had both BRAF V600E and V600K mutations and thus were excluded from either subgroup.

$\int$ Visceral disease was defined as that affecting the soft internal organs, including the lungs, heart, and the organs of the digestive, excretory, reproductive, and circulatory systems but excluding lymph nodes.

$\|$ The number of disease sites is the number of unique target and nontarget lesions, as defined by the Response Evaluation Criteria in Solid Tumors and identified by the investigator, not the number of metastases.

hazard ratio, 0.56 ; $95 \% \mathrm{CI}, 0.46$ to $0.69 ; \mathrm{P}<0.001$ ) (Fig. 2A). The hazard ratio for progression-free survival favored combination therapy in all subgroups (Fig 2B).
The objective response rate was 64\% (95\% CI, 59 to 69$)$ in the combination-therapy group versus $51 \%(95 \% \mathrm{CI}, 46$ to 57$)$ in the vemurafenib group $(\mathrm{P}<0.001)$ (Table 2$)$; the median duration 


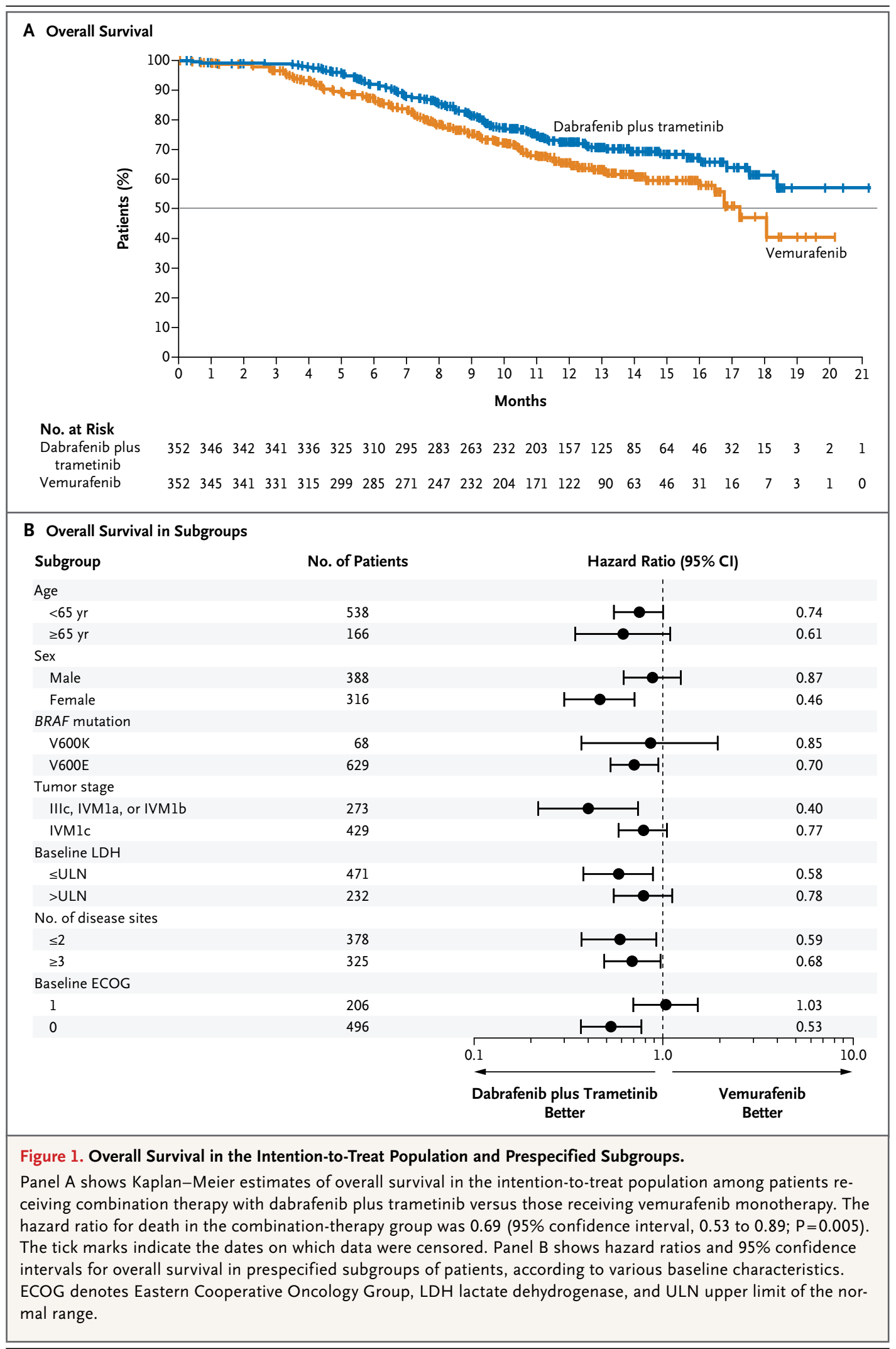

The New England Journal of Medicine

Downloaded from nejm.org at HAUPTBIBLIOTHEK UNIV ZUERICH on January 27, 2015. For personal use only. No other uses without permission. 


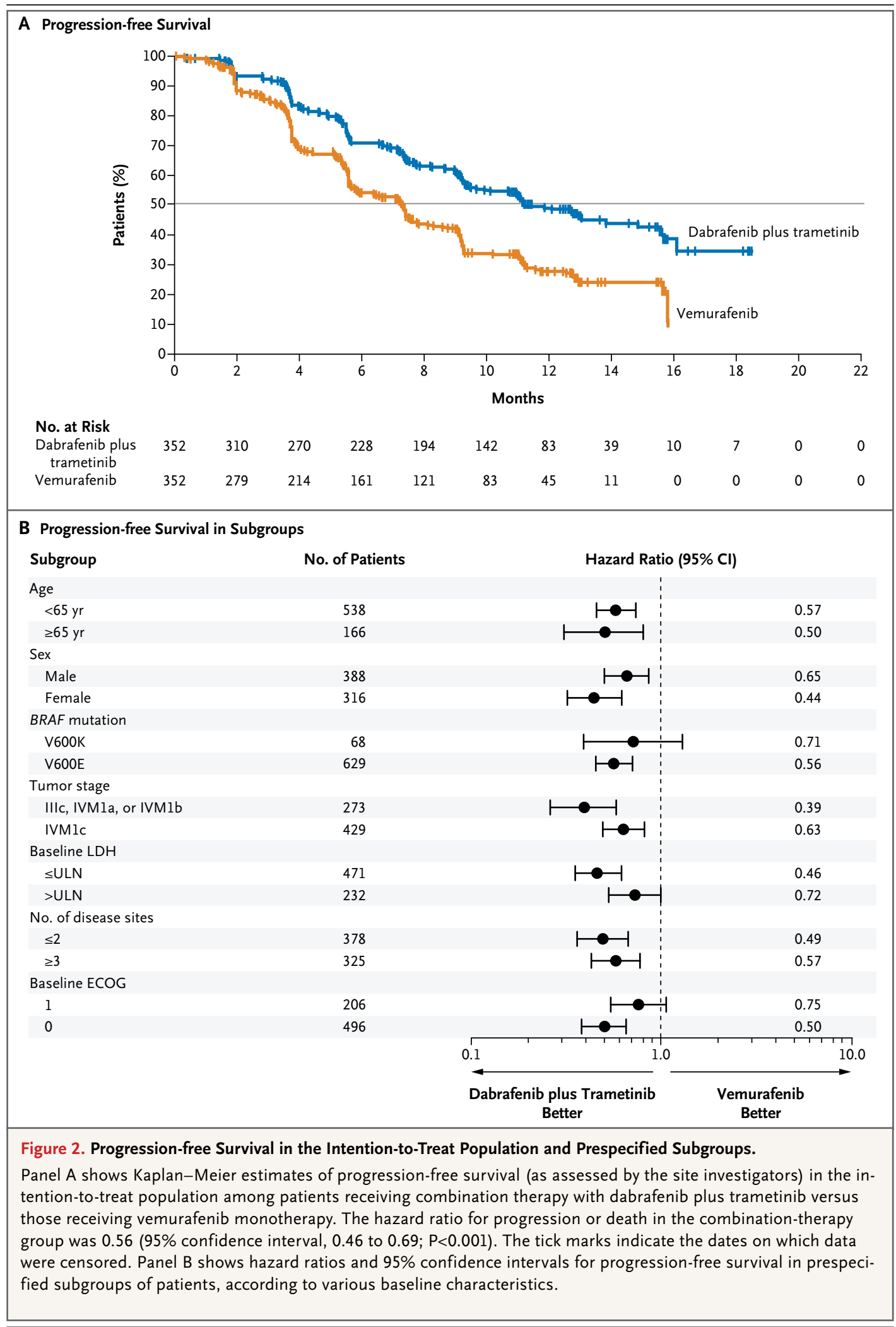

The New England Journal of Medicine 


\begin{tabular}{|lcc|}
\hline $\begin{array}{l}\text { Table 2. Investigator-Assessed Best Response (Intention-to-Treat } \\
\text { Population).* }\end{array}$ & $\begin{array}{c}\text { Dabrafenib } \\
\text { plus Trametinib } \\
\text { (N=351) }\end{array}$ & $\begin{array}{c}\text { Vemurafenib } \\
\text { (N=350) }\end{array}$ \\
$\begin{array}{l}\text { Response } \\
\text { Type of response - no. (\%) }\end{array}$ & $27(8)$ \\
$\quad$ Complete & $47(13)$ & $153(44)$ \\
Partial & $179(51)$ & $106(30)$ \\
Stable disease & $92(26)$ & $38(11)$ \\
Progressive disease & $22(6)$ & $26(7)$ \\
$\quad$ Not evaluated & $11(3)$ & $180(51)$ \\
Objective response rate & & $46.1-56.8$ \\
No. of patients with response (\%) $\dagger$ & $226(64)$ & $7.5(7.3-9.3)$ \\
$95 \% \mathrm{Cl}$ & $59.1-69.4$ & \\
Duration of response (95\% CI) - mo & $13.8(11.0-N R)$ &
\end{tabular}

* Data are missing for one patient in the combination-therapy group and two patients in the vemurafenib group because these patients did not have measurable disease at baseline. NR denotes not reached.

$\uparrow$ Included in the objective response are complete and partial responses. $\mathrm{P}<0.001$ for the between-group difference of $13 \%$ (95\% Cl, 6 to 20).

of response was 13.8 months (95\% CI, 11.0 to not reached) and 7.5 months (95\% CI, 7.3 to 9.3 ), respectively. In the combination-therapy group, a complete response was reported in 47 patients (13\%), as compared with 27 patients $(8 \%)$ in the vemurafenib group. The response rate for the BRAF V600E subgroup was similar to that in the overall population in both study groups: $64 \%$ in the combination-therapy group and $52 \%$ in the vemurafenib group; in the BRAF V600K subgroup, the response rates were $65 \%$ and $44 \%$, respectively.

After the discontinuation of study treatment, subsequent anticancer therapy was administered to $20 \%$ of the patients in the combination-therapy group and $43 \%$ of the patients in the vemurafenib group. The most common therapy after disease progression in the two groups was ipilimumab, which was administered to $12 \%$ of the patients in the combination-therapy group and $22 \%$ of the patients in the vemurafenib group. Post-trial therapies are listed in Table S3 in the Supplementary Appendix.

\section{ADVERSE EVENTS}

The safety analysis included the 699 patients who received at least one dose of a study drug (Table 3 , and Table S4 in the Supplementary Appendix). Most patients had adverse events that were deemed by the investigator to be related to the study treatment: $91 \%$ in the combination-therapy group and $98 \%$ in the vemurafenib group. The rates of permanent treatment discontinuation because of adverse events were similar (13\% and $12 \%$, respectively). The most common reasons for discontinuation were pyrexia and decreased ejection fraction (3\% for each event) in the combination-therapy group and arthralgia (2\%) in the vemurafenib group. Adverse events leading to dose reduction were reported in $33 \%$ of patients in the combination-therapy group and 39\% of those in the vemurafenib group; adverse events leading to dose interruption occurred in $55 \%$ and $56 \%$, respectively. Pyrexia was the most common reason for dose interruption (30\%) and dose reduction (14\%) in the combination-therapy group. Rash was the most common reason for dose interruption (14\%) and dose reduction (11\%) in the vemurafenib group.

The most frequent adverse events in the combination-therapy group were pyrexia (53\%), nausea $(35 \%)$, diarrhea $(32 \%)$, chills $(31 \%)$, fatigue (29\%), headache (29\%), and vomiting (29\%). In the vemurafenib group, the most frequent adverse events were arthralgia (51\%), rash $(43 \%)$, alopecia (39\%), diarrhea (38\%), nausea $(36 \%)$, and fatigue (33\%). Skin toxic effects were more frequent in the vemurafenib group than in the combination-therapy group, in particular rash $(43 \%$ vs. $22 \%)$, photosensitivity reaction ( $22 \%$ vs. $4 \%$ ), hand-foot syndrome ( $25 \%$ vs. $4 \%$ ), skin papillomas ( $23 \%$ vs. $2 \%)$, squamous-cell carcinomas and keratoacanthomas (18\% vs. $1 \%)$, and hyperkeratosis (25\% vs. $4 \%$ ). Pyrexia was more frequent in the combination-therapy group than in the vemurafenib group (53\% vs. $21 \%$ ).

Grade 3 or 4 adverse events occurred in 52\% of the patients in the combination-therapy group and in $63 \%$ of those in the vemurafenib group. Three fatal events occurred in each group; all were deemed by the investigator to be unrelated to the study drug. A total of 3 patients in the combination-therapy group died (2 patients from cerebral hemorrhage and 1 from brainstem hemorrhage), as did 3 in the vemurafenib group (1 patient each from acute coronary syndrome, cerebral ischemia, and pleural infection). Rates of noncutaneous cancers were similar in the two groups: 3 patients $(1 \%)$ in the combination-therapy group and $2(1 \%)$ in the vemurafenib group. A new primary melanoma was reported in $2(1 \%)$ in the combination-therapy group and 7 (2\%) in the vemurafenib group. An asymptomatic decrease in cardiac ejection fraction of grade 2 or 3 was reported in $8 \%$ of patients in the combination-therapy group and no 


\begin{tabular}{|c|c|c|c|c|}
\hline \multirow[t]{3}{*}{ Event } & \multicolumn{2}{|c|}{$\begin{array}{l}\text { Dabrafenib plus Trametinib } \\
\qquad(\mathrm{N}=350)\end{array}$} & \multicolumn{2}{|c|}{$\begin{array}{l}\text { Vemurafenib } \\
(N=349)\end{array}$} \\
\hline & Any Grade $†$ & Grade 3 & Any Grade & Grade 3 \\
\hline & \multicolumn{4}{|c|}{ number of patients (percent) } \\
\hline \multicolumn{5}{|l|}{$\begin{array}{l}\text { Clinically significant adverse events occurring } \\
\text { in } \geq 10 \% \text { of patients }\end{array}$} \\
\hline Any event & $343(98)$ & $167(48)$ & 345 (99) & $198(57)$ \\
\hline Pyrexiał & $184(53)$ & $15(4)$ & $73(21)$ & $2(1)$ \\
\hline Nausea & $121(35)$ & $1(<1)$ & $125(36)$ & $2(1)$ \\
\hline Diarrhea & $112(32)$ & $4(1)$ & $131(38)$ & $1(<1)$ \\
\hline Chills & $110(31)$ & $3(1)$ & $27(8)$ & 0 \\
\hline Vomiting & $101(29)$ & $4(1)$ & $53(15)$ & $3(1)$ \\
\hline Arthralgia & $84(24)$ & $3(1)$ & $178(51)$ & $15(4)$ \\
\hline Rash & $76(22)$ & $4(1)$ & $149(43)$ & $30(9)$ \\
\hline Alopecia & $20(6)$ & 0 & 137 (39) & $1(<1)$ \\
\hline Hand-foot syndrome $\mathbb{Z}$ & $14(4)$ & 0 & $87(25)$ & $1(<1)$ \\
\hline Hyperkeratosis & $15(4)$ & 0 & $86(25)$ & $2(1)$ \\
\hline Skin papilloma & $6(2)$ & 0 & $80(23)$ & $2(1)$ \\
\hline Photosensitivity reaction & $13(4)$ & 0 & $78(22)$ & $1(<1)$ \\
\hline \multicolumn{5}{|l|}{$\begin{array}{l}\text { Adverse events of interest occurring in }<10 \% \\
\text { of patients }\end{array}$} \\
\hline $\begin{array}{l}\text { Cutaneous squamous-cell carcinoma } \\
\text { (including keratoacanthoma) }\end{array}$ & $5(1)$ & $5(1)$ & $63(18)$ & $60(17)$ \\
\hline Decrease in ejection fraction & $29(8)$ & $13(4)$ & 0 & 0 \\
\hline Chorioretinopathy & $2(1)$ & 0 & $1(<1)$ & 0 \\
\hline Dermatitis acneiform & $22(6)$ & 0 & $20(6)$ & $4(1)$ \\
\hline
\end{tabular}

* Included in the safety analysis are 699 patients who received at least one dose of a study drug.

$\uparrow$ Grade 4 events occurred in 3 patients (with headache, asthenia, and an elevated aspartate aminotransferase level) in the combination-therapy group and in 5 patients (with hypertension, constipation, an elevated alanine aminotransferase level, and squamous-cell carcinoma) in the vemurafenib group. Grade 5 events occurred in 3 patients (with cerebral hemorrhage in 2 patients and brain-stem hemorrhage in 1 patient) in the combination-therapy group and in 3 patients (with acute coronary syndrome, cerebral ischemia, and pleural infection) in the vemurafenib group.

$†$ Pyrexia was defined as a body temperature of $38.5^{\circ} \mathrm{C}$ or more.

$\int$ The hand-foot syndrome includes the terms palmar-plantar erythrodysesthesia, plantar-palmar hyperkeratosis, and palmoplantar keratoderma.

patients in the vemurafenib group. Two grade 1 chorioretinopathy events were reported in the combination-therapy group and one in the vemurafenib group. In the vemurafenib group, grade 2 retinal-vein occlusion that was reported in 1 patient was considered to be related to the study drug; no retinal-vein occlusion was reported in the combination-therapy group.

\section{DISCUSSION}

In our study, patients with previously untreated metastatic melanoma with BRAF mutations who received combination therapy with dabrafenib and trametinib, as compared with vemurafenib monotherapy, had a relative reduction of $31 \%$ in the risk of death. Together with the previously reported phase 2 and phase 3 trials of dabrafenib plus trametinib, as compared with dabrafenib monotherapy, ${ }^{12,13}$ these data provide clear evidence for the benefit of this combination therapy over BRAF monotherapy in prolonging survival. Only modest activity has been observed with single-agent trametinib, or trametinib added to dabrafenib, in patients whose tumors have progressed while they were receiving dabrafenib monotherapy. ${ }^{17-19}$ Thus, our study shows that first-line use of both BRAF and MEK inhibitors 
resulted in higher response rates and longer durations of response, as well as delayed emergence of tumor resistance, which led to a significant overall survival benefit.

To evaluate a potential influence of subsequent anticancer therapies on the patients' survival, we analyzed the treatments that they received after progression in each group. More patients in the vemurafenib group received subsequent anticancer therapy than in the combination-therapy group (43\% vs. $20 \%$ ). The most common postprogression therapy in the two groups was ipilimumab, which is known to prolong survival in patients with metastatic melanoma. ${ }^{20,21}$ The fact that the median duration of exposure to vemurafenib was 4 months shorter than that for the combination therapy might partly explain why more patients in the vemurafenib group received postprogression therapy at this point. However, with more patients in the vemurafenib group having received a therapy that is known to affect overall survival, there is no evidence that differences in postprogression therapy contributed to the survival benefit seen in the combination-therapy group.

Post hoc subgroup analyses showed that the overall survival benefit was found in all prognostic subgroups except for patients with an ECOG score of 1 . The hazard ratio for these patients was 1.03, which is surprising, since other known prognostic factors, such as M1c stage, high LDH, and more than three sites of metastases, have hazard ratios that suggest a benefit for the combination therapy, as compared with vemurafenib. To understand the results in this subpopulation of patients, we looked at their subsequent therapies and conducted statistical tests for interactions with other prognostic factors. This observation did not appear to be driven by any specific factor and remains unexplained. Although no definitive conclusions can be drawn for this subgroup, a Cox model showed a hazard ratio for overall survival that was consistent with the results in the overall population, after accounting for the simultaneous effect of prognostic factors corrected for stratification factors.

Progression-free survival was also significantly prolonged among patients receiving the combination therapy, a finding that confirms the results of the COMBI-d phase 3 study, which evaluated progression-free survival in a similar population of patients who were treated with dabrafenib plus trametinib, as compared with dabrafenib alone. ${ }^{13,22}$ The results are also consistent with those of another study comparing vemurafenib plus cobimetinib with vemurafenib alone, which showed significant differences in progression-free survival of 9.9 and 6.2 months, respectively. ${ }^{14}$

The objective rate of tumor response was significantly higher in the combination-therapy group than in the vemurafenib group (64\% vs. $51 \%$, $\mathrm{P}<0.001)$, as was the duration of response, with a median duration of response in the combination-therapy group that was almost twice as long as that in the vemurafenib group (13.8 months vs. 7.5 months). Notably, the rate of complete response was also significantly higher in the combination-therapy group than in the vemurafenib group $(13 \%$ vs. $8 \%, \mathrm{P}=0.02)$ in a post hoc analysis.

The overall safety profile in the combinationtherapy group shows that this regimen has frequent low-grade toxicity. The incidences of grade 3 or 4 toxic effects and of adverse effects leading to dose reduction or treatment interruption were similar in the two study groups, with no deaths that were deemed to be related to the study drug. The spectrum of adverse effects differed in the two groups, with the most common cause for treatment modification reported as pyrexia in the combination-therapy group and rash in the vemurafenib group. Pyrexia is usually easily manageable with symptomatic treatment or transient interruption of dabrafenib or both dabrafenib and trametinib, with treatment reinitiation after the patient has been afebrile for a minimum of 24 hours. The education of both patients and physicians regarding pyrexia management is paramount to ensure that this side effect is not prolonged or complicated. A decreased ejection fraction was more frequent with the combination therapy than with vemurafenib ( $8 \%$ vs. $0 \%)$, and this side effect has been observed previously with single-agent MEK inhibitors. ${ }^{14,17}$

Skin adverse effects were less frequent in the combination-therapy group, especially the events that are linked to a paradoxical activation of the MAPK pathway, including both benign and malignant skin tumors. This finding is in accordance with preclinical models showing that the addition of MEK inhibitors may down-regulate the BRAF-inhibitor-induced paradoxical activation of the MAPK pathway. ${ }^{23-27}$ Thus, secondary resistance and paradoxical activation of the MAPK pathway that occur with BRAF-inhibitor 
monotherapy, which translate into rapid tumor relapses and emergence of skin cancers, respectively, were both improved by the combination therapy.

In conclusion, the combination of dabrafenib plus trametinib was superior to vemurafenib monotherapy with regard to all efficacy end points, including overall survival, with no additional overall toxicity.

Supported by GlaxoSmithKline.

Disclosure forms provided by the authors are available with the full text of this article at NEJM.org.

\section{APPENDIX}

The authors' affiliations are as follows: Gustave Roussy and INSERM Unité 981, Villejuif-Paris Sud (C.R.), Centre Hospitalier Universitaire de Reims-Hôpital Robert Debré, Reims (F.G.), and Centre Hospitalier Régional Universitaire de Lille-Hôpital Claude Huriez, Lille (L.M.) - all in France; Przychodnia Lekarska Komed, Konin (B.K.), Maria Sklodowska-Curie Memorial Cancer Center and Institute of Oncology, Warsaw (P.R.), Poznan University of Medical Sciences, Med-Polonia, Poznan (A.M.), and Swissmed Centrum Zdrowia and Medical University in Gdansk, Gdansk (K.D.) — all in Poland; Sheba Medical Center, Ramat, Israel (J.S.); Moscow City Oncology Hospital \#62 (D.S.) and Cancer Research Center (M.L.), Moscow; Universität Zürich, Zurich, Switzerland (R.D.); Istituto Oncologico Veneto Istituto Di Ricovero e Cura a Carattere Scientifico, Padua, Italy (V.C.-S.); Dermato-oncology Department, General University Hospital, Prague, Czech Republic (I.K.); Universitätsklinikum Schleswig-Holstein, Kiel (A.H.), and University Hospital Essen, Essen (D.S.) - both in Germany; Christie NHS Foundation Trust, Manchester (P.L.), and Mount Vernon Cancer Centre, Northwood (P.N.) — both in the United Kingdom; University Hospitals Leuven, Leuven Cancer Institute, Leuven, Belgium (P.W.); Melanoma Institute Australia, University of Sydney, Mater Hospital, Sydney (G.V.L.); Massachusetts General Hospital Cancer Center, Boston (K.F.); UCLA Jonsson Comprehensive Cancer Center, Los Angeles (A.R.); and GlaxoSmithKline Oncology Research and Development, Collegeville, PA (A.-M.M., P.S., W.C., J.L., S.D.R., S.M.L.).

\section{REFERENCES}

1. Chapman PB, Hauschild A, Robert C, et al. Improved survival with vemurafenib in melanoma with BRAF V600E mutation. N Engl J Med 2011;364:2507-16.

2. Hauschild A, Grob JJ, Demidov LV, et al. Dabrafenib in BRAF-mutated metastatic melanoma: a multicentre, openlabel, phase 3 randomised controlled trial. Lancet 2012;380:358-65.

3. Solit DB, Rosen N. Resistance to BRAF inhibition in melanomas. $\mathrm{N}$ Engl J Med 2011;364:772-4.

4. Shi H, Hugo W, Kong X, et al. Acquired resistance and clonal evolution in melanoma during BRAF inhibitor therapy. Cancer Discov 2014;4:80-93.

5. Van Allen EM, Wagle N, Sucker A, et al. The genetic landscape of clinical resistance to RAF inhibition in metastatic melanoma. Cancer Discov 2014;4:94-109.

6. Flaherty KT, Puzanov I, Kim KB, et al. Inhibition of mutated, activated BRAF in metastatic melanoma. $\mathrm{N}$ Engl J Med 2010;363:809-19.

7. Robert C, Arnault JP, Mateus C. RAF inhibition and induction of cutaneous squamous cell carcinoma. Curr Opin Oncol 2011;23:177-82.

8. Carnahan J, Beltran PJ, Babij C, et al. Selective and potent Raf inhibitors paradoxically stimulate normal cell proliferation and tumor growth. Mol Cancer Ther 2010;9:2399-410.

9. Oberholzer PA, Kee D, Dziunycz P, et al. RAS mutations are associated with the development of cutaneous squamous cell tumors in patients treated with RAF inhibitors. J Clin Oncol 2012;30:316-21.

10. Boussemart L, Routier E, Mateus C, et al. Prospective study of cutaneous sideeffects associated with the BRAF inhibitor vemurafenib: a study of 42 patients. Ann Oncol 2013;24:1691-7.
11. Su F, Viros A, Milagre C, et al. RAS mutations in cutaneous squamous-cell carcinomas in patients treated with BRAF inhibitors. N Engl J Med 2012;366:207-15. 12. Flaherty KT, Infante JR, Daud A, et al. Combined BRAF and MEK inhibition in melanoma with BRAF V600 mutations. N Engl J Med 2012;367:1694-703.

13. Long GV, Stroyakovskiy DL, Gogas H, et al. Combined BRAF and MEK inhibition versus BRAF inhibition alone in melanoma. N Engl J Med 2014;371:1877-88.

14. Larkin J, Ascierto PA, Dréno B, et al. Combined vemurafenib and cobimetinib in BRAF-mutated melanoma. $\mathrm{N}$ Engl J Med 2014;371:1867-76.

15. Eisenhauer EA, Therasse P, Bogaerts J, et al. New response evaluation criteria in solid tumours: revised RECIST guideline (version 1.1). Eur J Cancer 2009;45:228-47. 16. Oken MM, Creech RH, Tormey DC, et al. Toxicity and response criteria of the Eastern Cooperative Oncology Group. Am J Clin Oncol 1982;5:649-55.

17. Flaherty KT, Robert C, Hersey P, et al. Improved survival with MEK inhibition in BRAF-mutated melanoma. N Engl J Med 2012;367:107-14.

18. Menzies AM, Long GV. Dabrafenib and trametinib, alone and in combination for BRAF-mutant metastatic melanoma. Clin Cancer Res 2014;20:2035-43.

19. Kim KB, Kefford R, Pavlick AC, et al. Phase II study of the MEK1/MEK2 inhibitor trametinib in patients with metastatic BRAF-mutant cutaneous melanoma previously treated with or without a BRAF inhibitor. J Clin Oncol 2013;31:482-9.

20. Hodi FS, O'Day SJ, McDermott DF, et al. Improved survival with ipilimumab in patients with metastatic melanoma. N Engl J Med 2010;363:711-23. [Erratum, N Engl J Med 2010;363:1290.]
21. Robert C, Thomas L, Bondarenko I, et al. Ipilimumab plus dacarbazine for previously untreated metastatic melanoma. N Engl J Med 2011;364:2517-26.

22. Long GV, Stroyakovsky DL, Gogas H, et al. COMBI-d: a randomized, doubleblinded, Phase III study comparing the combination of dabrafenib and trametinib to dabrafenib and trametinib placebo as first-line therapy in patients (pts) with unresectable or metastatic BRAF V600E/K mutation-positive cutaneous melanoma. J Clin Oncol 2014;32:Suppl. abstract.

23. Corcoran RB, Dias-Santagata D, Bergethon K, Iafrate AJ, Settleman J, Engelman JA. BRAF gene amplification can promote acquired resistance to MEK inhibitors in cancer cells harboring the BRAF V600E mutation. Sci Signal 2010;3:ra84.

24. Villanueva J, Vultur A, Lee JT, et al. Acquired resistance to BRAF inhibitors mediated by a RAF kinase switch in melanoma can be overcome by cotargeting MEK and IGF-1R/PI3K. Cancer Cell 2010;18:683-95.

25. Greger JG, Eastman SD, Zhang V, et al. Combinations of BRAF, MEK, and PI3K/mTOR inhibitors overcome acquired resistance to the BRAF inhibitor GSK2118436 dabrafenib, mediated by NRAS or MEK mutations. Mol Cancer Ther 2012;11:909-20.

26. Emery CM, Vijayendran KG, Zipser $\mathrm{MC}$, et al. MEK1 mutations confer resistance to MEK and B-RAF inhibition. Proc Natl Acad Sci U S A 2009;106:20411-6.

27. Johannessen CM, Boehm JS, Kim SY, et al. COT drives resistance to RAF inhibition through MAP kinase pathway reactivation. Nature 2010;468:968-72.

Copyright (c) 2014 Massachusetts Medical Society. 